\title{
Musculoskeletal ultrasound abnormalities in patients on dialysis, compared to patients with pre-dialysis stage chronic kidney disease
}

\author{
Raluca-Daniela Decianu', Mihai Bojinca ${ }^{2,4}$, Cristina Capusa ${ }^{3,4}$, \\ Adrian Zugravu ${ }^{3,4}$, Violeta-Claudia Bojinca ${ }^{1,4}$ \\ ${ }^{1}$ Department of Internal Medicine and Rheumatology, "Sf. Maria" Clinical Hospital, Bucharest, Romania \\ 2Department of Internal Medicine and Rheumatology, "Ion Cantacuzino" Clinical Hospital, \\ Bucharest, Romania \\ ${ }^{3}$ Department of Nephrology, "Carol Davila” Clinical Hospital, Bucharest, Romania \\ 4"Carol Davila" University of Medicine and Pharmacy, Bucharest, Romania
}

\begin{abstract}
Background. The musculoskeletal system is deeply involved in patients with CKD on dialysis. The studies that were done by now with regarding this subject mostly aimed the bone metabolism. So far, there is few data on the subject concerning the articular and abarticular abnormalities in dialyzed patients(1).

Objectives. To identify the musculoskeletal soft tissue abnormalities associated to dialysis and to evaluate wheth-er or not they are more frequent. Also, we set ourselves to point out the importance of detecting these anomalies early in the evolution, in order to preserve the joint function and the quality of life, in a category of patients already severely affected.

Materials and methods. This is a prospective study, ran on a period of 4 months. We included 55 patients ( 32 in the study group - on dialysis, 23 in the control group - late stage pre-dialysis chronic kidney disease). For each patient, we evaluated 68 joints using ultrasonography. Also, a visual analogue scale for pain was applied for each patient.

Results. The findings vary from median nerve entrapment and tendon calcifications, to usual degenerative abnormalities, synovitis and tenosynovitis. The type of abnormalities does not vary between the two groups, but the percent of the affected patients was much higher in the study group.

Conclusions. A great number of patients from the study group presented important articular and mostly abarticular abnormalities, sometimes with no correlation with the algofunctional symptoms.
\end{abstract}

Keywords: musculoskeletal abnormalities, ultrasonography, chronic kidney disease, dialysis

\section{Abbreviations}

CKD - Chronic Kidney Disease

CKD-MBD - Chronic Kidney Disease-Mineral and Bone Disorder CRP - C Reactive Proteine

DIP - Distal InterPhangeal

ESR - Erythrocyte Sedimentation Rate

Ex - example

HD - HemoDialysis

MCP-MetaCarpoPhalangeal

MSK - MusculoSKeletal

\section{INTRODUCTION}

Late-stage CKD is one of the most frequent causes of morbidity and mortality worldwide, which leads to a great economic burden related to RRT (2). The higher the prevalence of CKD, the greater the importance of understanding the associated condi-

\author{
MTP - MetaTarsoPhalangeal \\ $\mathbf{N}$ - normal \\ PD - Peritoneal Dialysis \\ PIP - Proximal InterPhangeal \\ PTH - ParaTHormone \\ RRT - Renal Replacement Therapy \\ US -UltraSonography \\ VAS- Visual Analogue Scale
}

tions becomes, in order to personalize management approaches for this category of patients $(1,2)$.

It is a known fact that patients with late stage CKD and all the more so, patients on dialysis, have some particularities regarding the MSK abnormalities, such as an increased frequency and/or an earlier debut, in comparison to the general population $(3,4)$. 


\section{MATERIALS AND METHODS}

\section{Study design}

We elaborated a prospective study on 55 patients. On each patient was performed musculoskeletal ultrasonography, evaluating 68 joints, in two perpendicular planes. For the upper limb, the evaluated joints were: shoulder, elbow, wrist, MCP I-V, PIP I-V, DIP II-V, bilateral. For the lower limb, the evaluated joints were: hip, knee, ankle, MTP I-V, PIP I-V, DIP II-V, also bilateral.

All evaluations have been made by the same ultrasonographer, so there would be no inter-observer variations.

The ultrasonography machine used for examining all patients was a Samsung HM70A, from the equipment of the "Dr. Carol Davila" Clinical Hospital.

Also, for each patient, we applied a VAS for MSK pain or other type of discomfort (ex. paresthesia). We explained to each patient that they must relate only to the MSK symptoms and not to others, such as headaches or stomach pain.

\section{Patients and data}

All 55 patients are on a dialysis programme at the "Dr. Carol Davila" Clinical Hospital. The study group included 32 patients with CKD stage G5D (on dialysis), amongst whom 29 were on HD (3 sessions per week) and 3 on PD (hospitalized for an acute episode of the CKD). The control group included patients with late-stage CKD (G5 - eRFG $<15 \mathrm{ml} /$ $\min / 1.73 \mathrm{~m}^{2}$ ), in a pre-dialysis state.

The inclusion criteria were over 18 years of age, the informed consent for entering the study and at least 6 months on dialysis. The only exclusion criteria was the existence of an autoimmune rheumatic disease with articular or abarticular involvement, at the moment of the examination.

The laboratory evaluation included the renal status (creatinine, urea), PTH and inflammatory markers (ESR, CRP).

\section{RESULTS}

The spectre of abnormalities found, from the most to the least frequent, were median nerve entrapment within the carpal tunnel, tendinous calcifications, osteophytes, synovitis, tenosynovitis, bursitis, partial tendinous rupture and joint space narrowing (probably age related).

In the study group, the aspect of median nerve entrapment within the carpal tunnel was discovered in $66 \%$ of the patients, compared to $17 \%$ in the control group.

The tendinous calcifications were also found in $66 \%$ of the patients, compared to $43 \%$ in the control group. In most of these patients (63\%) the calcification were found in multiple sites. The most frequent unique location was the supraspinatus tendon at its insertion on the greater tuberosity (16\%), followed by the quadriceps femoris tendon at the insertion on the patella (9\%), the infraspinatus tendon, also inserted on the greater tuberosity $(6 \%)$ and the triceps tendon at its insertion on the olecranon process $(6 \%)$.

The VAS gives numeric results, which we divided in 3 groups: 0-3 (low), 4-6 (moderate) and 7-10 (high). In the study group, most patients presented low VAS results (41\%), 37\% declared moderate pain and/or paresthesia and only $22 \%$ accused a high lev-

TABLE 1. Baseline characteristics of the study population

\begin{tabular}{|c|c|c|c|c|}
\hline & Study group & & Control group & \\
\hline Sex distribution & M: 19 (59\%) & F: 13 (41\%) & M: 13 (57\%) & F: $10(43 \%)$ \\
\hline Age distribution (yrs) & 61,94 (31-81) & & 66,25 (41-84) & \\
\hline HD/PD & HD: 28 (87\%) & PD: 4 (13\%) & - & \\
\hline Time on dialysis (yrs) & $5,47(0,5-22)$ & & - & \\
\hline $\begin{array}{l}\text { Creatinine (mg/dl) } \\
\mathrm{N}: 0,6-1,2(\mathrm{M}) ; 0,5-1,1(\mathrm{~F})\end{array}$ & $8,44(1,08-11,73)$ & & $7,64(4,42-10,76)$ & \\
\hline $\begin{array}{l}\text { Urea (mg/dl) } \\
\mathrm{N}: 10-50\end{array}$ & $133,42(40-158)$ & & $120,29(46-169)$ & \\
\hline $\begin{array}{l}\text { Inorganic phosphate }(\mathrm{mg} / \mathrm{dl}) \\
\mathrm{N}: 2-5\end{array}$ & $6,18(4,2-7,7)$ & & $5,88(4,3-7,3)$ & \\
\hline $\begin{array}{l}\text { PTH (mg/dl) } \\
\text { N: } 15-68,3\end{array}$ & 616,03 (97-1509) & & 412,77 (103-978) & \\
\hline $\begin{array}{l}\text { CRP (mg/l) } \\
\mathrm{N}: 0-5\end{array}$ & $8,81(2-17)$ & & $11,74(3,5-21)$ & \\
\hline
\end{tabular}

$\mathrm{F}$ - female, $\mathrm{M}$ - male, yrs - years. 
el of pain and/orparesthesia. In the control group, the proportions of the 3 VAS groups are about the same. $54 \%$ of patients presented low VAS results, 32\% were in the moderate group and $14 \%$ declared a high level of pain and/or paresthesia.

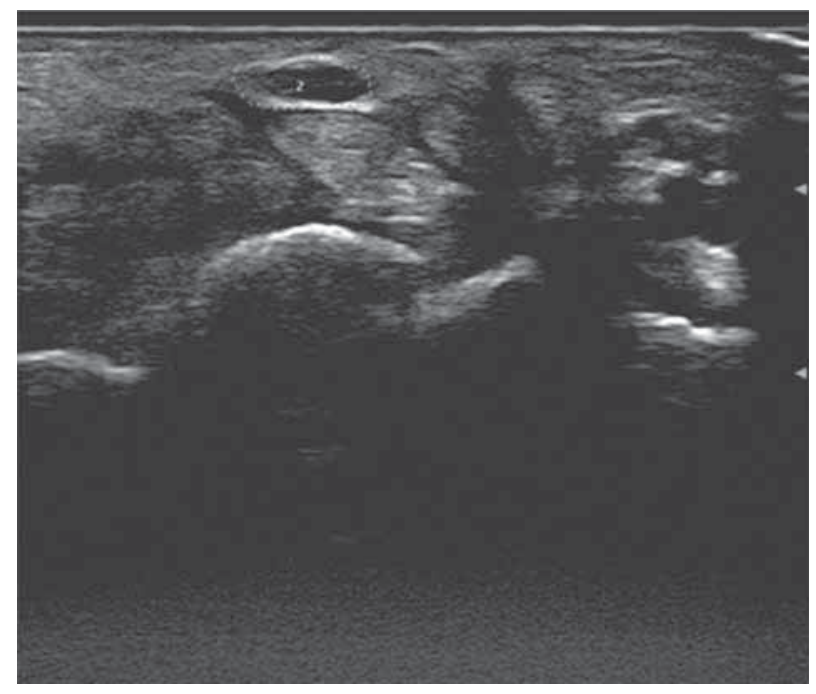

FIGURE 1. Median nerve enlargement in the carpal tunnel
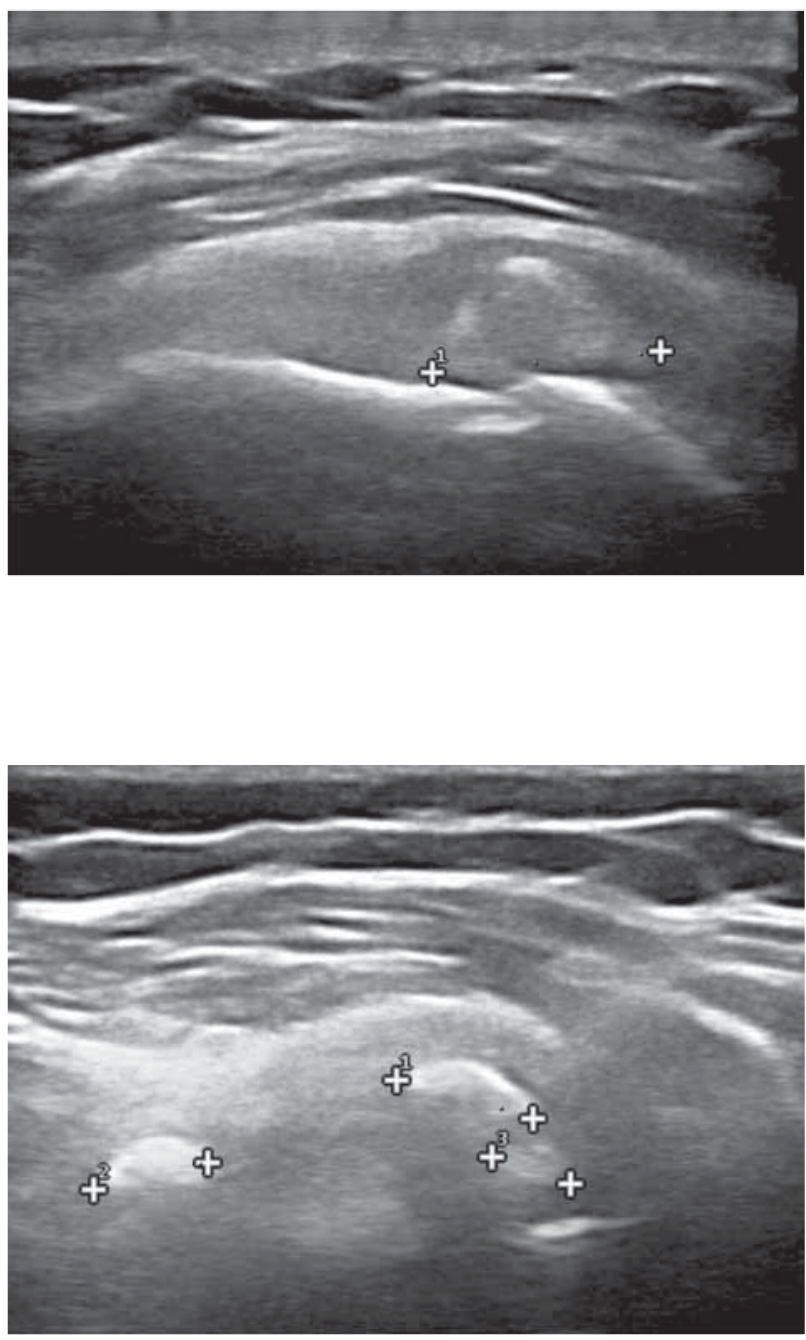

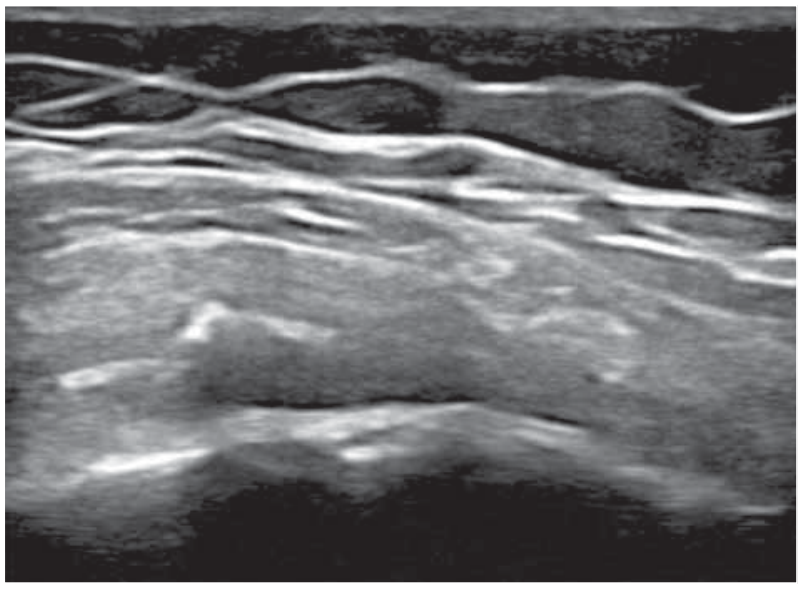

FIGURE 2,3,4. Calcifications of supraspinatus tendon

\section{DISCUSSIONS}

The percentage of US aspect of median nerve entrapment within the carpal tunnel was much higher in the study group (66\%), in comparison to the control group (17\%). Still, knowing that there are only $5 \%$ reported in the general population, it is clear that not only the dialysis process, but the CKD itself, by CKD-MBD, is responsible for these alterations in this category of patients $(5,6,7)$.

Setting as standard the percentage of tendinous calcifications in the general population (13-47\%), the results of our study situate the control group $(17 \%)$ at the same level as the general population, while the numbers in study group rise significantly over that percentage, up to $66 \%$ (8). It is known that the tendinous calcifications could also be age related $(8,9)$. The median age in our study is 64.09 , so one could say that some of the calcifications are age related. However, the percentage of calcifications is much higher in the study group than in the control group, although the median age in the study group (61.94) is even a little smallerthan in the control group (66.25). This proves that tendinous calcifications could be related either to a usually longer evolution of CKD-MBD in patients on dialysis, or to the process of dialysis itself.

The VAS results do not correspond to the high frequency of abnormalities found, so we couldn't help but wonder why. The answer to our question probably stands in the fact that CKD also causes uremic neuropathy. The prevalence of uremic neuropathy rises up to $83 \%$ in patients with CKD and it could prevent patients from feeling pain to its fullest (10).

\section{Study limitations}

In this phase of our study, we have a relatively small number of patients included, but we plan to 
keep evaluating more patients, in both groups. Also, in this phase of the study, we didn't separate the patients with diabetes in a different group, so some abnormalities might superpose.

The paraclinical evaluation was made during the last hospitalization (difference of 2 months at most), so the biomarkers might vary at the moment of the examination.

The personal history was declarative or taken from the file from the last hospitalization (in patients lacking medical documents, also declarative), so there could be cases of undeclared MSK diseases.

\section{CONCLUSIONS}

CKD-MBD lead to ectopic calcifications, not only vascular, but with multiple other locations, such as tendons.

The MSK abnormalities detected in this study lead to pain, paresthesia, limited function of the joints and altered quality of life.

The treatment options for these alterations and for the pain they cause are limited in CKD, but these patients could benefit from kinetotherapyor from other rehabilitation techniques (11). In order for this benefit to be greater, the alterations must be discovered as early as possible (12).

This is a first step to elaborating a programme of medical gymnastics, which the patients could benefit from during the long hours of the dialysis sessions.

It is a known fact that sports and physical activities in general reduce the risk of depression, so this type of programme could also influence the depression rate, which reaches very high levels among patients on dialysis $(13,14)$.

\section{Acknowledgements}

The authors would like to thank the management and the physicians from the "Carol Davila" Nephrology Clinical Hospital, Bucharest, for providing access to patients on dialysis programme and also to the ultrasonography machine.

Conflict of interest: none declared Financial support: none declared

9. Hussein D.A., El-Azizi N.O., Meged A.H.A. et al. Ultrasonographic Tendon Alteration in Relation to Parathyroid Dysfunction in Chronic Hemodialysis Patients. Clin Med Insights Arthritis MusculoskeletDisord. 2015; 8: 9-14.

10. Wittmann I., Stirban A., Tesfaye S. et al. Neuropathy in chronic kidney disease. Diabetes, Stoffwechsel und Herz. 2015; 24(4): 251-255.

11. Katayama A., Miyatake N., Nishi H. Evaluation of physical activity and its relationship to health-related quality of life in patients on chronic hemodialysis. Environ Health Prev Med. 2014; 19: 380.

12. Bessa B., de Oliveira Leal V., Moraes C. et al. Resistance Training in Hemodialysis Patients: A Review. RehabilNurs. 2015; 40(2): 111-126.

13. Farrokhi F., Abedi N., Beyene J. et al. Association Between Depression and Mortality in Patients Receiving Long-term Dialysis: A Systematic Review and Meta-analysis. Elsevier. 2014; 63(4):623635.

14. Lopes A.A., Lantz B., Morgenstern H. et al. Associations of SelfReported Physical Activity Types and Levels with Quality of Life, Depression Symptoms, and Mortality in Hemodialysis Patients: The DOPPS. Clin J Am SocNephrol. 2014;9(10):1702-1712. 